\title{
Hypernuclear structure with the new Nijmegen potentials
}

\author{
I. Vidaña, A. Polls, A. Ramos, and H.-J. Schulze \\ Departament d'Estructura i Constituents de la Matèria, Universitat de Barcelona, Av. Diagonal 647, E-08028 Barcelona, Spain
}

(Received 10 March 2001; published 11 September 2001)

\begin{abstract}
We perform continuous-choice Brueckner-Hartree-Fock calculations of hypernuclear matter, using the recent Nijmegen potentials for hyperon-nucleon and hyperon-hyperon interactions. Single-particle observables of the various hyperons in bulk matter, as well as properties of single- and double-lambda hypernuclei, employing an extended Skyrme-Hartree-Fock scheme, are presented. We find that the potentials tend to overbind the single hypernuclei and strongly underbind the double hypernuclei.
\end{abstract}

DOI: 10.1103/PhysRevC.64.044301 PACS number(s): 21.80.+a, 21.65.+f, 21.10.Dr, 21.30.Fe

\section{INTRODUCTION}

For the past couple of years, aside from nonrelativistic nucleon-nucleon potentials of very high quality, hyperonnucleon potentials [1-6], that are consistent with the existing data set on elastic hyperon-nucleon scattering, have also become available for the theoretical modeling of hypernuclei and hypermatter. Recently, a new set of potentials was presented [7] that also involves, besides the usual hyperonnucleon force in the strangeness $S=-1$ channel, extrapolations to the hyperon-hyperon $(S=-2,-3,-4)$ channels.

Since, unfortunately, at present the data set on hyperonnucleon scattering is rather scarce and of relatively poor quality compared to the nucleon-nucleon case, the corresponding potentials are not very well constrained, allowing for considerable freedom in their construction. We therefore take in this article the opportunity to employ these new potentials for microscopical calculations of hypernuclear structure. The motivation is to judge their quality by confrontation with data of finite hypernuclei, where available. We will therefore present results for single-particle levels of singlelambda hypernuclei, on which quite detailed experimental information exists, as well as discuss the main features of infinite hypermatter with the main relevance for astrophysical applications [8].

The situation is especially uncertain for the hyperonhyperon channels, for which no scattering information is available at all, and the new potentials, with no additional parameters and obeying an approximate SU(3) symmetry, constitute therefore mere "extrapolations" to the $S=-2$, $-3,-4$ sectors. Regarding this aspect, the only possible confrontation with reality at the moment appears the comparison with information on the potentially observed (three) doublelambda hypernuclei ${ }_{\Lambda \Lambda}^{6} \mathrm{He},{ }_{\Lambda \Lambda}^{10} \mathrm{Be}$, and ${ }_{\Lambda \Lambda}^{13} \mathrm{~B}$ [9]; and this is one of the objectives of this paper.

The degree of freedom in the construction of the potentials is evidenced by the fact that in Ref. [7] actually six different potential parametrizations were given, which fit equally well the scattering data, but produce different scattering lengths in the $\Lambda N$ and $\Sigma N$ channels. In this article we present results that are obtained with the potentials " $A$ " and " $F$ " of that reference and compare with results using the "old" Nijmegen soft-core potential [3], denoted " $O$ " in the following.

\section{FORMALISM}

The results we present in this paper are based on generalized Brueckner-Hartree-Fock (BHF) calculations (employing continuous single-particle potentials in the computation of the $G$ matrices) of lambda-hypermatter, i.e., symmetric nuclear matter of density $\rho_{N}$ containing a fraction of lambda hyperons with density $\rho_{\Lambda}$.

The technicalities of these calculations are explained in detail in Refs. [10,11], and will not be repeated here. We only mention that the principal difficulties are caused by the choice of continuous single-particle potentials for all types of particles involved, as well as by the presence of a large number of coupled channels. This renders the computations very time-consuming. In the following we will make use of the principal results of the calculations, which are the binding energy per baryon as function of the various (in our case nucleon and lambda) partial densities, $B / A\left(\rho_{N}, \rho_{\Lambda}\right)$, as well as the momentum-dependent single-particle potentials of all types of particles involved, $U_{q}(k)$, where the index $q$ $=n, p, \Lambda, \Sigma^{-0+}, \Xi^{-0}$ labels the different baryons that we take into account.

We will further below present results on certain features of these bulk matter quantities, but also use them as input information for the determination of properties of hypernuclei in an extended Skyrme-Hartree-Fock (SHF) model [12]. This model was recently employed together with the "old" Nijmegen hyperon-nucleon potential, and quite satisfactory results for the properties of single-lambda hypernuclei were obtained. A comparison with the equivalent predictions involving the new potentials is therefore of interest. More importantly, contrary to the old potentials, the new ones involve extensions to the hyperon-hyperon sector and allow therefore to make also predictions for double-lambda hypernuclei, which we will explore as well.

In Ref. [12] a hyperon-hyperon interaction was not present, so that we introduce briefly in the following the slightly extended scheme involving such a force. For a more detailed account the reader is referred to Ref. [12]. The local energy density functional of hypermatter depends on the densities $\rho_{q}=\sum_{i=1}^{N_{q}}\left|\phi_{q}^{i}\right|^{2}$ and kinetic energy densities $\tau_{q}$ $=\sum_{i=1}^{N_{q}}\left|\nabla \phi_{q}^{i}\right|^{2}$ and is written as $\epsilon=\epsilon_{N}+\epsilon_{\Lambda}$, where $\epsilon_{N}$ is the purely nucleonic SHF functional, as specified in Ref. [13], and 


$$
\epsilon_{\Lambda}=\frac{1}{2 m_{\Lambda}} \tau_{\Lambda}+\epsilon_{N \Lambda}\left(\rho_{N}, \rho_{\Lambda}\right)
$$

is the functional accounting for the presence of lambdas, which is due to the action of hyperon-nucleon and hyperonhyperon forces. It can be constructed from the BHF results for $B / A$ as

$$
\begin{aligned}
\epsilon_{N \Lambda}\left(\rho_{N}, \rho_{\Lambda}\right)= & \left(\rho_{N}+\rho_{\Lambda}\right) \frac{B}{A}\left(\rho_{N}, \rho_{\Lambda}\right)-\rho_{N} \frac{B}{A}\left(\rho_{N}, 0\right) \\
& -\frac{3}{5} \frac{C}{2 m_{\Lambda}} \rho_{\Lambda}^{5 / 3},
\end{aligned}
$$

where the last term corresponds to the kinetic energy contribution of the lambdas. The constant $C=\left(3 \pi^{2}\right)^{2 / 3} \approx 9.571$ has been introduced. However, as in Ref. [12], we prefer to work with a Schrödinger equation that involves, instead of the bare lambda mass $m_{\Lambda}$, the hyperon effective mass $m_{\Lambda}^{*}$, as extracted from the BHF single-particle potential

$$
\frac{m_{\Lambda}^{*}}{m_{\Lambda}}=\left[1+\frac{U_{\Lambda}\left(k_{F}^{(\Lambda)}\right)-U_{\Lambda}(0)}{k_{F}^{(\Lambda) 2} / 2 m}\right]^{-1} .
$$

For this purpose the energy density functional is written instead as

$$
\epsilon_{\Lambda}=\frac{1}{2 m_{\Lambda}^{*}} \tau_{\Lambda}+\epsilon_{N \Lambda}\left(\rho_{N}, \rho_{\Lambda}\right)-\left(\frac{m_{\Lambda}}{m_{\Lambda}^{*}}-1\right) \frac{3}{5} \frac{C}{2 m_{\Lambda}} \rho_{\Lambda}^{5 / 3} .
$$

Minimizing the total energy of the hypernucleus, $E$ $=\int d^{3} r \epsilon(r)$, one arrives with Eq. (4) at the SHF Schrödinger equation

$$
\begin{aligned}
& {\left[-\nabla \cdot \frac{1}{2 m_{q}^{*}(r)} \nabla+V_{q}(r)-i W_{q}(r)(\nabla \times \boldsymbol{\sigma})\right] \phi_{q}^{i}(r)} \\
& \quad=-e_{q}^{i} \phi_{q}^{i}(r),
\end{aligned}
$$

with the wave functions $\phi_{q}^{i}$ and the single-particle energies $-e_{q}^{i}$ for the different single-particle levels $i$ and species $q$ $=n, p, \Lambda$. The SHF mean fields are

$$
\begin{gathered}
V_{N}=V_{N}^{\mathrm{SHF}}+\frac{\partial \epsilon_{N \Lambda}}{\partial \rho_{N}}+\frac{\partial}{\partial \rho_{N}}\left(\frac{m_{\Lambda}}{m_{\Lambda}^{*}\left(\rho_{N}\right)}\right)\left(\frac{\tau_{\Lambda}}{2 m_{\Lambda}}-\frac{3}{5} \frac{C}{2 m_{\Lambda}} \rho_{\Lambda}^{5 / 3}\right), \\
V_{\Lambda}=\frac{\partial \epsilon_{N \Lambda}}{\partial \rho_{\Lambda}}-\left(\frac{m_{\Lambda}}{m_{\Lambda}^{*}\left(\rho_{N}\right)}-1\right) \frac{C}{2 m_{\Lambda}} \rho_{\Lambda}^{2 / 3},
\end{gathered}
$$

where $V_{N}^{\mathrm{SHF}}$ is the nucleonic Skyrme mean field without hyperons and $W_{N}$ the nucleonic spin-orbit mean-field, as given in Ref. [13]. At the present level of approximation we do not include a spin-orbit force for the lambda, which is justified by the experimental observation of very small spin-orbit splittings.
TABLE I. Parameters of the functional representations of energy density and lambda effective mass, Eqs. (7) and (8), for the potentials $O, A$, and $F$.

\begin{tabular}{lrrr}
\hline \hline & $O$ & $A$ & $F$ \\
\hline$\alpha_{1}$ & 327 & 423 & 384 \\
$\alpha_{2}$ & 1159 & 1899 & 1473 \\
$\alpha_{3}$ & 1163 & 3795 & 1933 \\
$\alpha_{4}$ & 335 & 577 & 635 \\
$\alpha_{5}$ & 1102 & 4017 & 1829 \\
$\alpha_{6}$ & 1660 & 11061 & 4100 \\
$\alpha_{7}$ & 0 & 38 & 50 \\
$\alpha_{8}$ & 0 & 186 & 545 \\
$\alpha_{9}$ & 0 & 22 & 981 \\
\hline$\mu_{1}$ & 1 & 0.98 & 0.93 \\
$\mu_{2}$ & 1.83 & 1.72 & 2.19 \\
$\mu_{3}$ & 5.33 & 3.18 & 3.89 \\
$\mu_{4}$ & 6.07 & 0 & 0 \\
\hline \hline
\end{tabular}

As in Ref. [12], we provide parametrizations of the numerical results for the key quantities $\epsilon_{N \Lambda}$ and $m_{\Lambda}^{*}$ in the following functional forms $\left(\rho_{N}\right.$ and $\rho_{\Lambda}$ given in units of $\mathrm{fm}^{-3}, \epsilon_{N \Lambda}$ in $\left.\mathrm{MeV} \mathrm{fm}^{-3}\right)$ :

$$
\begin{aligned}
\epsilon_{N \Lambda}\left(\rho_{N}, \rho_{\Lambda}\right) \approx & -\left(\alpha_{1}-\alpha_{2} \rho_{N}+\alpha_{3} \rho_{N}^{2}\right) \rho_{N} \rho_{\Lambda} \\
& +\left(\alpha_{4}-\alpha_{5} \rho_{N}+\alpha_{6} \rho_{N}^{2}\right) \rho_{N} \rho_{\Lambda}^{5 / 3} \\
& -\left(\alpha_{7}-\alpha_{8} \rho_{\Lambda}+\alpha_{9} \rho_{\Lambda}^{2}\right) \rho_{\Lambda}^{2},
\end{aligned}
$$

$$
\frac{m_{\Lambda}^{*}}{m_{\Lambda}}\left(\rho_{N}\right) \approx \mu_{1}-\mu_{2} \rho_{N}+\mu_{3} \rho_{N}^{2}-\mu_{4} \rho_{N}^{3} .
$$

The parameters $\alpha_{i}$ and $\mu_{i}$ are listed in Table I for the three different potentials $O, A$, and $F$ that we use.

The functional form of Eq. (8) is purely phenomenological, whereas that of Eq. (7) is guided by the fact that the energy density functional can be related to the BHF singleparticle potentials in the following manner [12]:

$$
\begin{aligned}
\epsilon_{N \Lambda}\left(\rho_{N}, \rho_{\Lambda}\right)= & \sum_{k<k_{F}^{(\Lambda)}}\left[2 U_{\Lambda}^{(N)}(k)+U_{\Lambda}^{(\Lambda)}(k)\right] \\
& +2 \sum_{k<k_{F}^{(N)}}\left[\left.U_{N}^{(N)}(k)\right|_{\rho_{\Lambda}}-\left.U_{N}^{(N)}(k)\right|_{\rho_{\Lambda}=0}\right],
\end{aligned}
$$

where $U_{A}^{(B)}$ denotes the BHF single-particle potential component of baryon $A$ due to the presence of baryons $B$ in the medium [10]. In extension to Ref. [12], the last term on the right-hand side of Eq. (7) and $U_{\Lambda}^{(\Lambda)}(k)$ in Eq. (9) are due to the presence of hyperon-hyperon interactions. We remark that nevertheless we still keep a simple parametrization of the lambda effective mass, Eq. (8), depending only on the 


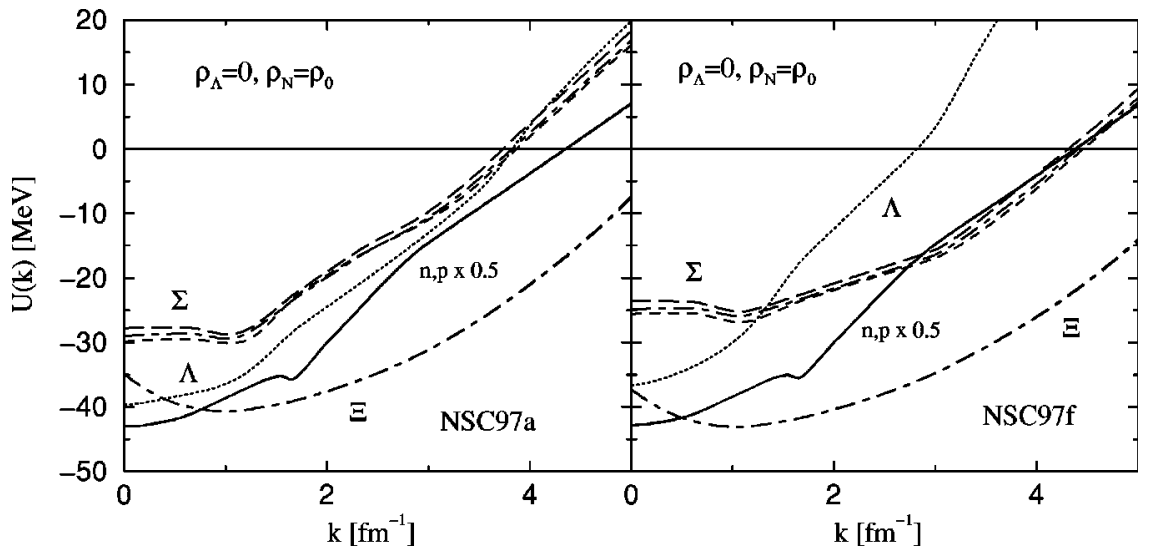

FIG. 1. Hyperon and nucleon single-particle potentials in pure nucleonic matter $\left(\rho_{\Lambda}=0\right)$ at saturation density $\left(\rho_{N}=\rho_{0}=0.17\right.$ $\left.\mathrm{fm}^{-3}\right)$. The nucleonic curves are scaled down by a factor of $1 / 2$. The upper dashed curve pertains to $\Sigma^{0}$, the middle one to $\Sigma^{+}$, and the lower one to $\Sigma^{-}$. Results with the potentials $A$ (left panel) and $F$ (right panel) are compared. nucleon density. This still reproduces the numerical results relatively well and we consider it sufficient at the present stage of approximation.

\section{RESULTS}

\section{A. Bulk matter}

We begin with a presentation of the principal properties of infinite hypermatter in our model: In order to illustrate the basic features, Fig. 1 shows the complete set of nucleon and hyperon single-particle potentials in pure nuclear matter at saturation density $\left(\rho_{N}=\rho_{0}=0.17 \mathrm{fm}^{-3}, \rho_{Y}=0\right)$ resulting from our calculations. The hyperon single-particle potentials are much less attractive than the nucleonic ones, reflecting the weaker strength of the hyperon-nucleon compared to the nucleon-nucleon potentials. Concerning the effective masses $m^{*} / m$, indicated by the curvature of the single-particle potentials, those of the lambda are generally smaller than unity, $m_{\Sigma}^{*} / m_{\Sigma}$ is very close to unity, and $m_{\Xi}^{*} / m_{\Xi}$ larger than unity.

Compared to previous BHF calculations employing the much simpler gap-choice prescription for the single-particle potentials $[7,11,14,15]$, our new results indicate only small changes for the lambda, but substantially more binding for the sigma hyperons. With respect to the potential $O$ [10], the new potentials predict more binding for the lambda and in particular for the sigma hyperons. More detailed comparisons between the new and old potentials will be given in the following.

The most interesting features that can be extracted from plots like Fig. 1 are the hyperon "well depths" $U_{Y}(k=0),{ }^{1}$ as well as effective masses, as defined in Eq. (3) for the lambda. The corresponding results are displayed in Fig. 2 as a function of nucleonic density and the values at normal density are also listed in Table II. One notes that the new potentials predict relatively strong attraction for all types of hyperons, which at least in the case of the sigma and the cascade hyperons appears not to be supported by the present experimental information. In the case of the $\Xi$ hyperons, early analysis of old emulsion data [16] obtained an attrac-

\footnotetext{
${ }^{1}$ It should be noted, however, that these quantities are slightly more attractive than the SHF potentials $V_{Y}$, due to rearrangement contributions to the latter, see Ref. [12].
}

tive $\Xi$-nucleus well potential of around $-24 \mathrm{MeV}$, while recent $\left(K^{-}, K^{+}\right)$spectra on ${ }_{\Lambda}^{12} \mathrm{C}$, obtained at $\mathrm{KEK}$ and $\mathrm{BNL}$ [17] and analyzed within the distorted wave Born approximation, favor a shallower potential of around $-14 \mathrm{MeV}$. With respect to the $\Sigma$ hyperon, data on $\Sigma^{-}$atoms seemed to be compatible with attractive potentials of around $-25 \mathrm{MeV}$ at $\rho_{0}[18,19]$, while more recent fits including data of heavier atoms suggested a repulsive potential in the nuclear interior [20-22]. A recent comparison of $\left(K^{-}, \pi^{ \pm}\right)$ spectra calculated in plane wave impulse approximation [23] with data taken at BNL [24] also seems to favor a repulsive $\Sigma$-nucleus interaction, although more sophisticated treatments including the distortions of the incoming and outgoing mesons would be desirable before drawing definite conclusions.

The differences in binding between the three sigma hyperons in symmetric nuclear matter are in the case of the new

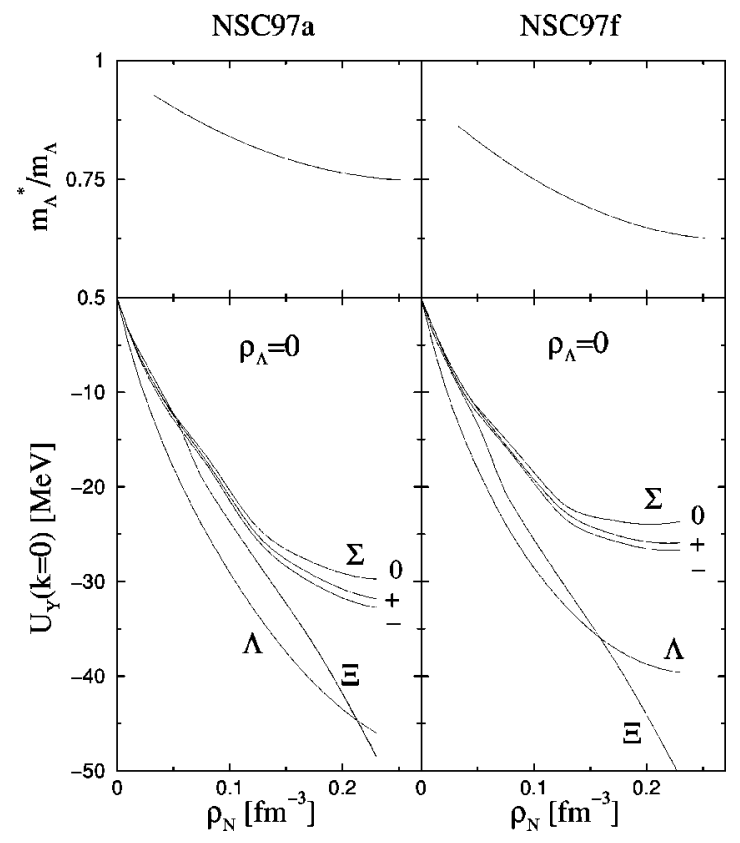

FIG. 2. Lambda effective mass (top panels) and well depths of the different hyperons (bottom panels) as functions of nucleon density in pure nucleonic matter $\left(\rho_{\Lambda}=0\right)$. Results obtained with the potentials $A$ and $F$ are shown in the left and right panel, respectively. 
TABLE II. Depth of the different hyperon single-particle potentials, $-U_{Y}(k=0)$ (in $\mathrm{MeV}$ ), in nuclear matter of normal density $\rho_{N}=\rho_{0}=0.17 \mathrm{fm}^{-3}$, evaluated with different potentials.

\begin{tabular}{lrcc}
\hline \hline$Y$ & $O$ & $A$ & $F$ \\
\hline$\Lambda$ & 30.0 & 39.7 & 36.6 \\
$\Sigma^{-}$ & 8.0 & 29.7 & 25.5 \\
$\Sigma^{0}$ & 15.2 & 27.6 & 23.5 \\
$\Sigma^{+}$ & 8.7 & 28.8 & 24.8 \\
$\Xi^{-}$ & & 35.1 & 37.3 \\
$\Xi^{0}$ & & 35.1 & 37.3 \\
\hline \hline
\end{tabular}

potentials solely generated by the different masses, whereas the potential $O$ involves explicit isospin breaking in the different sigma-nucleon channels [3]. We also note that the strongly repulsive Lane (isovector) term, which is responsible for the existence of a bound ${ }_{\Sigma}^{4} \mathrm{He}$ state [25,26], comes out to be opposite in sign for the new Nijmegen models [23]. The properties of lambdas will be discussed in more detail below.

Concerning the lambda effective mass, we obtain at saturation density the values $m_{\Lambda}^{*} / m_{\Lambda} \approx 0.78,0.67$, and 0.81 for the potentials $A, F$, and $O$, respectively. Due to the strength of the new potentials and a repulsive $p$-wave contribution [15], the corresponding values are slightly smaller than phenomenological ones reported in Ref. [27].

After reviewing the properties of individual hyperons in pure nuclear matter, we come now to the discussion of systems with finite strangeness fraction. In this article we will restrict to the most relevant case of lambda hypermatter characterized by partial densities $\rho_{N}$ and $\rho_{\Lambda}$, or equivalently, total density $\rho=\rho_{N}+\rho_{\Lambda}$ and lambda fraction $x_{\Lambda}=\rho_{\Lambda} / \rho$.

A particular important aspect of lambda hypermatter is the formation of cascade hyperons via the $\Lambda \Lambda \rightarrow N \Xi$ reaction, once a threshold of the lambda fraction is reached. Hypernuclei beyond a certain strangeness fraction contain therefore necessarily other types of hyperons besides lambdas [2830]. Quantitatively, the lambda threshold density is determined by the equation

$$
\mu_{\Xi}+\mu_{N}-2 \mu_{\Lambda}+\Delta m=0,
$$

where $\mu_{q}=\partial \epsilon / \partial \rho_{q}$ are the chemical potentials of the different species (depending on the different partial densities) and $\Delta m=m_{\Xi}+m_{N}-2 m_{\Lambda} \approx 23 \mathrm{MeV}$, is the relevant difference of rest masses of the baryons involved. Due to the fact that this quantity is quite small, the onset of $\Xi$ hyperons can in principle take place at relatively low strangeness fraction. It depends crucially on the in-medium properties (chemical potentials) of the three species $N, \Lambda$, and $\Xi$.

The most relevant information is the lambda fraction $x_{\Lambda}$ corresponding to the onset of $\Xi$ formation in hypermatter with partial nucleon density $\rho_{N} \approx \rho_{0}$, since this resembles the situation in heavy hypernuclei. For this purpose we can write

$$
\mu_{N}\left(\rho_{0}, \rho_{\Lambda}\right)=\frac{B}{A}\left(\rho_{0}, 0\right)+\frac{\partial \epsilon_{N \Lambda}}{\partial \rho_{N}}\left(\rho_{0}, \rho_{\Lambda}\right),
$$

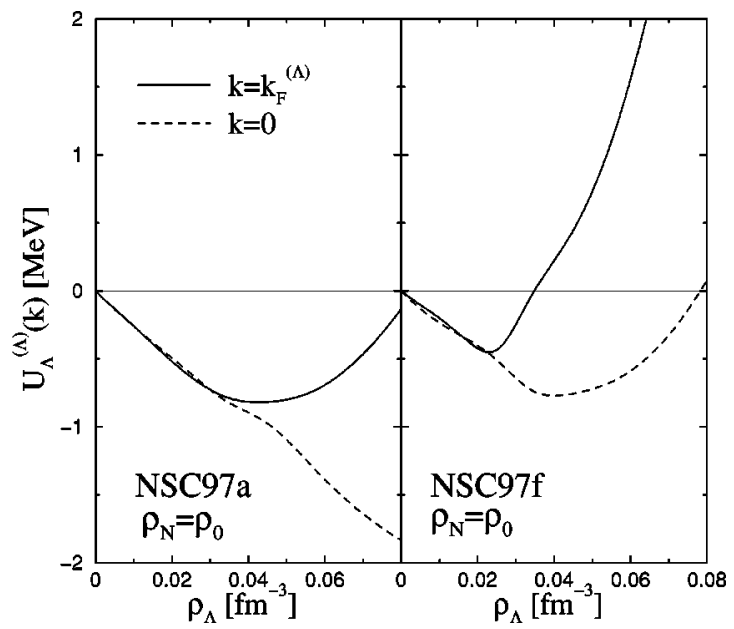

FIG. 3. BHF single-particle potential $U_{\Lambda}^{(\Lambda)}(k)$ as a function of lambda density for fixed $\rho_{N}=\rho_{0}=0.17 \mathrm{fm}^{-3}$ and two momenta $k$ $=0, k_{F}^{(\Lambda)}$.

$$
\begin{gathered}
\mu_{\Lambda}\left(\rho_{0}, \rho_{\Lambda}\right)=\frac{C}{2 m_{\Lambda}} \rho_{\Lambda}^{2 / 3}+\frac{\partial \epsilon_{N \Lambda}}{\partial \rho_{\Lambda}}\left(\rho_{0}, \rho_{\Lambda}\right), \\
\mu_{\Xi}\left(\rho_{0}, \rho_{\Lambda}\right) \approx U_{\Xi}\left(\rho_{0}, 0\right),
\end{gathered}
$$

using the fact that at nucleonic saturation density we have $\mu_{N}\left(\rho_{0}\right)=B / A\left(\rho_{0}\right) \approx-16 \mathrm{MeV}$, and approximating the $\Xi$ chemical potential by the BHF well depth. The terms involving partial derivatives of $\epsilon_{N \Lambda}$ can now easily be calculated from Eq. (7), and solving Eq. (10) we obtain the following values for the lambda fraction at which $\Xi$ hyperons will start to form: $x_{\Lambda}=0.17,0.15$, and 0.08 for the potentials $O, A$, and $F$, respectively. The value for the potential $O$ has been obtained with the assumption $U_{\Xi}=-15 \mathrm{MeV}$ and is therefore higher than those for the new potentials, which yield very large (but probably unrealistic) values for the binding of the $\Xi$ in nuclear matter, although there is some compensation, since also the lambda is more bound with these potentials. In any case, the phenomenon of $\Xi$ formation is not relevant for hypernuclei containing only a small number of lambdas, which will be discussed in the following.

In view of the relevance for double-lambda hypernuclei, we display finally in Fig. 3 the BHF single-particle potential component due to the lambda-lambda interaction, $U_{\Lambda}^{(\Lambda)}(k)$, as a function of lambda density for fixed $\rho_{N}=\rho_{0}$ and for two typical momenta $k=0, k_{F}^{(\Lambda)}$. Estimating very roughly the typical lambda density in a double-lambda hypernucleus as $\rho_{\Lambda} \approx 2 \rho_{0} / A \approx 0.02 \mathrm{fm}^{-3}$ for ${ }_{\Lambda \Lambda}^{10} \mathrm{Be}$, one can expect from the figure typical two-lambda bond energies of not more than $+0.5 \mathrm{MeV}$ (attractive) for the potential $A$ and even less for the potential $F$. This will be confirmed later by our microscopical calculation; however, it can already be stated here that clearly the theoretical value is far too small, compared to the experimental estimate of about $5 \mathrm{MeV}$ [9].

\section{B. Single and double-lambda hypernuclei}

After presenting the principal features of infinite hypermatter, we come now to the modelling of hypernuclei within 


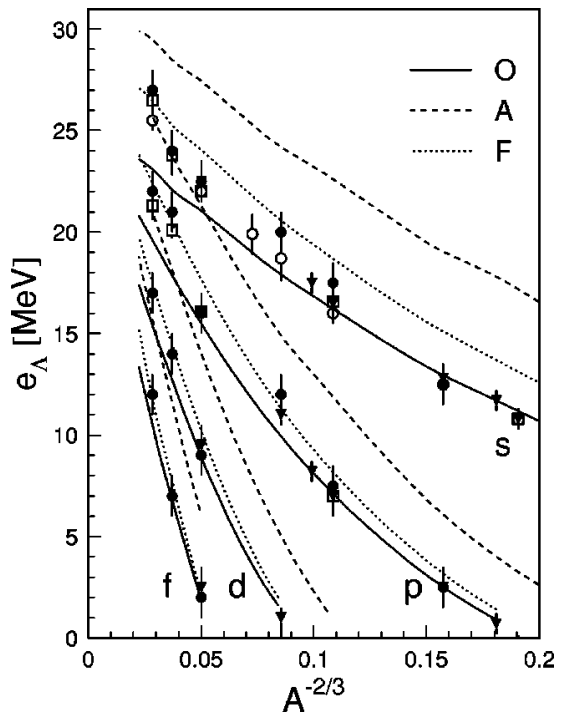

FIG. 4. Lambda single-particle energies for different hypernuclei as a function of mass number $A$. The lines indicate theoretical results obtained with different potentials, and the markers show experimental data from Refs. [31,32].

the Skyrme-Hartree-Fock approach that utilizes these results as input quantities, as explained in the previous section.

The most significant results are the lambda single-particle levels of various single-lambda hypernuclei, for which experimental results are available for comparison [31,32]. In Ref. [12], using the potential $O$, our model predicted a slight underbinding of heavy hypernuclei. As we have seen, the new potentials produce more binding in infinite matter, which is also reflected in our results for hypernuclei: The lambda single-particle levels $e_{\Lambda}^{i}(i=1 s, 1 p, 1 d, 1 f)$ are displayed in Fig. 4 and listed in Table III, which compares the values obtained with the different potentials. Indeed, consistent with the results in infinite matter, the new potentials yield significantly more binding. Even taking into account the theoretical uncertainties involved in our model, it seems that the potential $A$ can be excluded, while the potential $F$ gives actually good results for the heaviest nuclei, however systematically overbinds the light nuclei by about $2 \mathrm{MeV}$. The rearrangement corrections to $e_{\Lambda}^{i}$ are repulsive and of the order of $1 \mathrm{MeV}$ for light nuclei, decreasing smoothly with $A$. They improve the agreement of the potential $F$ with the ex-
TABLE IV. Bond energies $\Delta B_{\Lambda \Lambda}$ (in MeV), Eq. (12), of several double-lambda hypernuclei.

\begin{tabular}{lccc}
\hline \hline & $O$ & $A$ & $F$ \\
\hline${ }_{\Lambda \Lambda} \mathrm{Be}$ & -0.34 & 0.37 & -0.35 \\
${ }_{\Lambda \Lambda}^{14} \mathrm{C}$ & -0.41 & 0.32 & -0.47 \\
${ }_{\Lambda \Lambda}^{18} \mathrm{O}$ & -0.41 & 0.32 & -0.41 \\
${ }_{\Lambda \Lambda}^{30} \mathrm{Si}$ & -0.33 & 0.25 & -0.35 \\
${ }_{\Lambda \Lambda}^{42} \mathrm{Ca}$ & -0.31 & 0.19 & -0.32 \\
${ }_{9 \Lambda} \mathrm{Zr}$ & -0.21 & 0.09 & -0.24 \\
${ }_{\Lambda \Lambda}^{14} \mathrm{Ce}$ & -0.14 & 0.05 & -0.18 \\
${ }_{\Lambda \Lambda}^{210} \mathrm{~Pb}$ & -0.12 & 0.01 & -0.15 \\
\hline \hline
\end{tabular}

perimental results. As stated in Ref. [12], the main contribution to the rearrangement correction is provided by the correct treatment of the center of mass.

Proceeding now to double-lambda hypernuclei, we list in Table IV the bond energy

$$
\Delta B_{\Lambda \Lambda}=2 E\left({ }_{\Lambda}^{A-1} Z\right)-E\left({ }_{\Lambda \Lambda}^{A} Z\right)-E\left({ }^{A-2} Z\right)
$$

evaluated with the different potentials. Whereas the old Nijmegen potential contains no hyperon-hyperon interaction and yields small negative bond energies due to rearrangement effects of the core (see the discussion in Ref. [12]), the new potentials do contain such forces and could in principle be able to overcome this effect and to reproduce the experimental values $\Delta B_{\Lambda \Lambda} \approx 5 \mathrm{MeV}$, indicating a strong $\Lambda \Lambda$ attraction. In practice, however, the binding is still grossly underestimated by about a factor 10 with the potential $A$, whereas the potential $F$ predicts even a slightly repulsive effect. One can relate these results to the BHF single-particle potential component $U_{\Lambda}^{(\Lambda)}$, displayed in Fig. 3. Assuming for simplicity a momentum independent $U_{\Lambda}^{(\Lambda)}$ (which is well fulfilled for very low lambda density), one arrives, using Eqs. (9) and (12), at

$$
\Delta B_{\Lambda \Lambda} \approx U_{\Lambda}^{(\Lambda)}\left(\bar{\rho}_{\Lambda}\right)-U_{\Lambda}^{(\Lambda)}\left(2 \bar{\rho}_{\Lambda}\right) \approx-U_{\Lambda}^{(\Lambda)}\left(\bar{\rho}_{\Lambda}\right),
$$

where $\bar{\rho}_{\Lambda} \approx \rho_{0} / A$ is a rough estimate of the average density of lambdas in a single-lambda hypernucleus. Indeed, comparing the values given in Table IV and in Fig. 3, the relation Eq. (13) is approximately fulfilled for the potential $A$,

TABLE III. Lambda single-particle levels (in MeV) for different single-lambda hypernuclei. Predictions of the potentials $O, A$, and $F$ are

\begin{tabular}{|c|c|c|c|c|c|c|c|c|c|c|c|c|c|c|c|c|c|c|c|c|}
\hline & \multicolumn{4}{|c|}{$1 s$} & \multicolumn{4}{|c|}{$1 p$} & \multicolumn{4}{|c|}{$1 d$} & \multicolumn{4}{|c|}{$1 f$} & \multicolumn{4}{|c|}{$1 g$} \\
\hline & $O$ & $A$ & $F$ & Expt. & $O$ & $A$ & $F$ & Expt. & $O$ & $A$ & $F$ & Expt. & $O$ & $A$ & $F$ & Expt. & $O$ & $A$ & $F$ & Expt. \\
\hline$\left({ }_{\Lambda}^{13} \mathrm{C}\right)$ & 11.7 & 17.8 & 13.7 & (11.7) & 0.9 & 4.0 & 1.4 & (0.7) & & & & & & & & & & & & \\
\hline$\left({ }_{\Lambda}^{16} \mathrm{O}\right)$ & 13.3 & 19.2 & 15.5 & (12.5) & 3.0 & 6.7 & 3.7 & (2.5) & & & & & & & & & & & & \\
\hline$\left({ }_{\Lambda}^{28} \mathrm{Si}\right)$ & 16.4 & 22.8 & 18.9 & (17.5) & 7.4 & 12.2 & 8.5 & (7.5) & & 1.3 & & & & & & & & & & \\
\hline${ }_{\Lambda}^{41} \mathrm{Ca} \quad\left({ }_{\Lambda}^{40} \mathrm{Ca}\right)$ & 18.0 & 24.3 & 20.7 & (20.0) & 10.1 & 15.1 & 11.5 & (12.0) & 1.6 & 5.3 & 2.0 & (1.0) & & & & & & & & \\
\hline$\left.{ }_{\Lambda}^{91} \mathrm{Zr} \quad{ }_{\Lambda}^{89} \mathrm{Y}\right)$ & 21.1 & 27.5 & 24.1 & (22.5) & 15.6 & 21.4 & 17.8 & $(16.0)$ & 9.1 & 14.2 & 10.4 & (9.0) & 2.1 & 6.3 & 2.4 & (2.0) & & & & \\
\hline${ }_{\Lambda}^{141} \mathrm{Ce}\left({ }_{\Lambda}^{139} \mathrm{La}\right)$ & 22.1 & 28.4 & 25.3 & (24.0) & 17.9 & 23.8 & 20.5 & (21.0) & 12.8 & 18.2 & 14.5 & (14.0) & 6.9 & 11.7 & 7.8 & (7.0) & 0.6 & 4.7 & 0.6 & (1.0) \\
\hline${ }_{\Lambda}^{209} \mathrm{~Pb} \quad\left({ }_{\Lambda}^{208} \mathrm{~Pb}\right)$ & 23.1 & 29.5 & 26.5 & (27.0) & 19.6 & 25.7 & 22.4 & (22.0) & 15.4 & 21.0 & 17.5 & (17.0) & 10.5 & 15.7 & 11.8 & (12.0) & 5.1 & 9.7 & 5.6 & (7.0) \\
\hline
\end{tabular}
compared with experimental data from Ref. [32] (in brackets) with errors of about $\pm 1 \mathrm{MeV}$. 
whereas the $\Lambda \Lambda$ interaction in the potential $F$ is so weak and turning into repulsion at higher momenta, that the results obtained are very close to those of the potential $O$ without any $\Lambda \Lambda$ interaction.

In summary, clearly much more binding in the lambdalambda interaction is required in order to give satisfactory results, even if our model is only quite schematic, neglecting any correlations beyond the mean field, which could be quite important in particular for light nuclei [33]. A related conclusion was drawn in Ref. [7], where very small lambda-lambda scattering lengths were obtained with the new potentials.

Since the new potentials fail completely already for the treatment of double hypernuclei, we will in this work not discuss the speculative properties of multihypernuclei.

\section{CONCLUSIONS}

We have in this article confronted the recent Nijmegen hyperon-nucleon and hyperon-hyperon potentials with experimental information on single- and double-lambda hypernuclei. Our theoretical framework involved an extended BHF scheme, using continuous single-particle spectra for all species, and, based on these results, a SHF model of hypernuclei.

Even taking into account the theoretical uncertainties of our model (like the lack of three-baryon forces and finiterange effects $[12,27,34])$, the results obtained are not very satisfactory: With the new potentials all types of hyperons are too strongly bound in nuclear matter; the single-lambda hypernuclei (in particular light ones) are overbound, whereas the resulting effective lambda-lambda interaction is by far too weak compared to the values deduced experimentally.

Clearly a readjustment of the potential parameters in particular for the hyperon-hyperon channels is necessary and simple extrapolations guided by $\mathrm{SU}(3)$ symmetry are not sufficient, as had actually already been pointed out in the original article [7].

\section{ACKNOWLEDGMENTS}

We thank J. Cugnon, A. Lejeune, and M. Hjorth-Jensen for helpful discussions. This work was supported in part by the programs "Estancias de científicos y tecnólogos extranjeros en España," SGR98-11 (Generalitat de Catalunya), and DGICYT (Spain) No. PB98-1247.
[1] M.M. Nagels, Th. Rijken, and J.J. de Swart, Phys. Rev. D 15, 2547 (1977).

[2] M.M. Nagels, Th. Rijken, and J.J. de Swart, Phys. Rev. D 20, 1633 (1979).

[3] P. Maessen, Th. Rijken, and J.J. de Swart, Phys. Rev. C 40, 2226 (1989).

[4] A. Reuber, K. Holinde, and J. Speth, Nucl. Phys. A570, 543 (1994).

[5] T. Fujita, Y. Fujiwara, C. Nakamoto, and Y. Suzuki, Prog. Theor. Phys. 100, 931 (1998).

[6] K. Tominaga, T. Ueda, M. Yamaguchi, N. Kijima, D. Okamoto, K. Miyagawa, and T. Yamada, Nucl. Phys. A642, 483 (1998).

[7] Th.A. Rijken, V.G.J. Stoks, and Y. Yamamoto, Phys. Rev. C 59, 21 (1999); V.G.J. Stoks and Th.A. Rijken, ibid. 59, 3009 (1999).

[8] M. Baldo, G.F. Burgio, and H.-J. Schulze, Phys. Rev. C 58, 3688 (1998); 61, 055801 (2000); I. Vidaña, A. Polls, A. Ramos, L. Engvik, and M. Hjorth-Jensen, ibid. 62, 035801 (2000)

[9] M. Danysz et al., Phys. Rev. Lett. 11, 20 (1963); Nucl. Phys. 49, 121 (1963); J. Prowse, Phys. Rev. Lett. 17, 782 (1966); R.H. Dalitz, D.H. Davis, P.H. Fowler, A. Montwill, J. Pniewski, and J.A. Zakrzewski, Proc. R. Soc. London, Ser. A 426, 1 (1989); S. Aoki et al., Prog. Theor. Phys. 85, 1287 (1991); C.B. Dover, D.J. Millener, A. Gal, and D.H. Davis, Phys. Rev. C 44, 1905 (1991); K. Imai, Nucl. Phys. A547, 199c (1992); D.H. Davis, ibid. A547, 369c (1992); G.B. Franklin, ibid. A585, 83c (1995).

[10] H.-J. Schulze, M. Baldo, U. Lombardo, J. Cugnon, and A. Lejeune, Phys. Rev. C 57, 704 (1998); Phys. Lett. B 355, 21 (1995)

[11] I. Vidaña, A. Polls, A. Ramos, M. Hjorth-Jensen, and V.G.J.
Stoks, Phys. Rev. C 61, 025802 (2000).

[12] J. Cugnon, A. Lejeune, and H.-J. Schulze, Phys. Rev. C 62, 064308 (2000).

[13] D. Vautherin and D.M. Brink, Phys. Rev. C 5, 626 (1972); M. Beiner, H. Flocard, Nguyen Van Giai, and P. Quentin, Nucl. Phys. A238, 29 (1975).

[14] V.G.J. Stoks and T.-S. Lee, Phys. Rev. C 60, 024006 (2000).

[15] Y. Yamamoto, S. Nishizaki, and T. Takatsuka, Prog. Theor. Phys. 103, 981 (2000).

[16] C.B. Dover and A. Gal, Ann. Phys. (N.Y.) 146, 309 (1983).

[17] T. Fukuda et al., Phys. Rev. C 58, 1306 (1998); P. Khaustov et al., ibid. 61, 054603 (2000).

[18] C.J. Batty et al., Phys. Lett. 74B, 27 (1978); 87B, 324 (1979).

[19] E. Oset, P. Fernández de Córdoba, L.L. Salcedo, and R. Brockmann, Phys. Rep. 188, 79 (1990).

[20] C.J. Batty, E. Friedman, and A. Gal, Phys. Lett. B 335, 273 (1994).

[21] J. Mareš, E. Friedman, A. Gal, and B.K. Jennings, Nucl. Phys. A594, 311 (1995).

[22] C.J. Batty, E. Friedman, and A. Gal, Phys. Rep. 287, 385 (1997).

[23] J. Da̧browski, Phys. Rev. C 60, 025205 (1999).

[24] R. Sawafta, Nucl. Phys. A639, 103c (1998); S. Bart et al., Phys. Rev. Lett. 83, 5238 (1999).

[25] T. Harada, S. Shinmura, Y. Akaishi, and H. Tanaka, Nucl. Phys. A507, 715 (1990); T. Harada, ibid. A672, 181 (2000).

[26] T. Nagae et al., Phys. Rev. Lett. 80, 1605 (1998).

[27] Q.N. Usmani and A.R. Bodmer, Phys. Rev. C 60, 055215 (1999).

[28] J. Schaffner-Bielich and A. Gal, Phys. Rev. C 62, 034311 (2000).

[29] J. Schaffner, C. Greiner, and H. Stöcker, Phys. Rev. C 46, 322 (1992); J. Schaffner, C.B. Dover, A. Gal, C. Greiner, D.J. Mil- 
lener, and H. Stöcker, Ann. Phys. (N.Y.) 235, 35 (1994).

[30] J. Žofka et al., Phys. Lett. B 235, 25 (1990); A.J. Baltz et al., ibid. 325, 7 (1994); P. H. Pile, in Properties and Interactions of Hyperons, edited by B. J. Gibson, P. D. Barnes, and K. Nakai (World Scientific, Singapore, 1994).

[31] B. Povh, Nucl. Phys. A335, 233 (1980); R.E. Chrien, ibid. A478, 705c (1988); P.H. Pile, Phys. Rev. Lett. 66, 2585
(1991); T. Hasegawa et al., Phys. Rev. C 53, 1210 (1996).

[32] S. Ajimura et al., Nucl. Phys. A585, 173c (1995).

[33] A.R. Bodmer and Q.N. Usmani, Nucl. Phys. A468, 653 (1987); S.B. Carr, I.R. Afnan, and B.F. Gibson, ibid. A625, 143 (1997).

[34] F. Arias de Saavedra, G. Co', and A. Fabrocini, Phys. Rev. C 63, 064308 (2001). 